\title{
GROUPS OF HEIGHT FOUR
}

\author{
ALFRED W. HALES \\ (Received 15 March; revised 28 September 1979) \\ Communicated by $\mathbf{H}$. Lausch
}

\begin{abstract}
If $G$ and $H$ are infinite groups then $G$ is said to be larger than $H(H \leqslant G)$ if there are subgroups $A$ of $G, B$ of $H$, each of finite index, such that $B$ is an epimorphic image of $A$. Pride (1979) showed that if $G$ has finite 'height' with respect to the quasi-order $\leqslant$ then there are only finitely many (classes of) minimal groups $H$ with $H \leqslant G$, and asked whether this were true without the minimality restriction on $H$. This paper gives a negative answer to his question by exhibiting a group $G$ of height four with infinitely many (classes of) groups $H$ satisfying $H \leqslant G$.
\end{abstract}

1980 Mathematics subject classification (Amer. Math. Soc.): 20 E 99, 20 K 15.

\section{Introduction}

Pride (1976) defined a quasi-order of the class of infinite groups as follows: If $G$ and $H$ are infinite groups, then $G$ is larger than $H(H \leqslant G)$ if there are subgroups $A$ of $G, B$ of $H$, each of finite index, such that $B$ is an epimorphic image of $A$. Then $G$ and $H$ are said to be equally large $(G \simeq H)$ if each is larger than the other, and $\preccurlyeq$ induces a partial order on the $\simeq$ classes of infinite groups. Groups $G$ lying in minimal classes under this partial order are said to have height one and, more generally, a group has height $n$ if $n$ is maximal such that there exists a sequence $G_{1} \preccurlyeq G_{2} \preccurlyeq \ldots \preccurlyeq G_{n}=G$ of infinite groups no two of which are equally large.

Pride (1979) showed that if $G$ has finite height then there are only finitely many (up to $\simeq$ ) height one groups $H$ with $H \preccurlyeq G$. He then asked whether this result was still true without the minimality restriction on $H$. In this paper we give a negative answer to his question by exhibiting a group $G$ of height four such that there are infinitely many (up to $\simeq$ ) $H$ of height three with $H \preccurlyeq G$. 


\section{Construction}

The group we construct will in fact be abelian. Hence, from now on, we assume all groups under consideration to be abelian and use additive notation.

Let $\mathbf{Z}$ and $\mathbf{Q}$ denote the additive groups of integers and rationals, respectively. Fix a prime $p$ and let $p^{-\infty} \mathbf{Z}$ denote the subgroup of $\mathbf{Q}$ consisting of those fractions whose denominators are powers of $p$. Then the quasicyclic quotient group $p^{-\infty} \mathbf{Z} / \mathbf{Z}$ is usually denoted by $\mathbf{Z}\left(p^{\infty}\right)$. There is a natural action of the $p$-adic integers $\mathbf{Z}_{p}$ on $\mathbf{Z}\left(p^{\infty}\right)$ which we write as left multiplication. Let $\alpha, \beta$ be $p$-adic integers and define a map $\varphi_{\alpha, \beta}$ from $\mathbf{Z}\left(p^{\infty}\right)$ to $\mathbf{Z}\left(p^{\infty}\right) \oplus \mathbf{Z}\left(p^{\infty}\right) \oplus \mathbf{Z}\left(p^{\infty}\right)$ by $\varphi_{\alpha, \beta}(x)=x \oplus \alpha x \oplus \beta x$. Then $\varphi_{\alpha, \beta}\left(\mathbf{Z}\left(p^{\infty}\right)\right)$ is a subgroup of $\mathbf{Z}\left(p^{\infty}\right) \oplus \mathbf{Z}\left(p^{\infty}\right) \oplus \mathbf{Z}\left(p^{\infty}\right)$ isomorphic to $\mathbf{Z}\left(p^{\infty}\right)$. But the quotient group $\left(p^{-\infty} \mathbf{Z} \oplus p^{-\infty} \mathbf{Z} \oplus p^{-\infty} \mathbf{Z}\right) /(\mathbf{Z} \oplus \mathbf{Z} \oplus \mathbf{Z})$ is naturally isomorphic to $\mathbf{Z}\left(p^{\infty}\right) \oplus \mathbf{Z}\left(p^{\infty}\right) \oplus \mathbf{Z}\left(p^{\infty}\right)$. Hence, by the isomorphism theorems, $\varphi_{\alpha, \beta}\left(\mathbf{Z}\left(p^{\infty}\right)\right)$ corresponds to a subgroup of $p^{-\infty} \mathbf{Z} \oplus p^{-\infty} \mathbf{Z} \oplus p^{-\infty} \mathbf{Z}$ containing $\mathbf{Z} \oplus \mathbf{Z} \oplus \mathbf{Z}$. We denote this subgroup by $G(\alpha, \beta)$. Then we have:

THEOREM. (a) For all $\alpha, \beta$ in $\mathbf{Z}_{p}, G(\alpha, \beta)$ has height four.

(b) If $\alpha, \beta$ are algebraically independent over $\mathbf{Q}$ then there are infinitely many (up to $\simeq$ ) $H$ of height three with $H \preccurlyeq G(\alpha, \beta)$.

Before proceeding to the proof, observe that $G(\alpha, \beta)$ can alternatively be described as the set of all $x \oplus y \oplus z$ in $p^{-\infty} \mathbf{Z} \oplus p^{-\infty} \mathbf{Z} \oplus p^{-\infty} \mathbf{Z}$ such that $\alpha x-y$ and $\beta x-z$ are $p$-adic integers. Furthermore, if we denote by $G(\alpha)$ the subgroup of $p^{-\infty} \mathbf{Z} \oplus p^{-\infty} \mathbf{Z}$ obtained by projecting $G(\alpha, \beta)$ onto its first two 'coordinates', then $G(\alpha)$ is the set of all $x \oplus y$ such that $\alpha x-y$ is a $p$-adic integer. $G(\alpha)$ is, of course, the subgroup of $p^{-\infty} \mathbf{Z} \oplus p^{-\infty} \mathbf{Z}$ corresponding to the image of the map $\varphi_{\alpha}: \mathbf{Z}\left(p^{\infty}\right) \rightarrow \mathbf{Z}\left(p^{\infty}\right) \oplus \mathbf{Z}\left(p^{\infty}\right)$ given by $\varphi_{\alpha}(x)=x \oplus \alpha x$.

\section{Proof}

(a) It is easy to see that $G(\alpha, \beta)$ has height at least four from the sequence

$$
\mathbf{Z}\left(p^{\infty}\right) \preccurlyeq p^{-\infty} \mathbf{Z} \preccurlyeq G(\alpha) \preccurlyeq G(\alpha, \beta) .
$$

Here we consider $G(\alpha, \beta)$ as mapped onto $G(\alpha)$ by projection onto its first two coordinates and $G(\alpha)$ as mapped onto $p^{-\infty} Z$ by projection onto its first coordinate. Furthermore $\mathbf{Z}\left(p^{\infty}\right)$ is an epimorphic image of $p^{-\infty} \mathbf{Z}$. To see that no two of these groups are equally large note that their torsion-free ranks are $0,1,2$ and 3 respectively, and that $H \preccurlyeq G$ with $H, G$ abelian implies that the torsion-free rank of $G$ is at least as big as that of $H$. 
Now suppose that $G(\alpha, \beta)$ has height bigger than four, so that there is a sequence of infinite groups

$$
G_{1} \preccurlyeq G_{2} \preccurlyeq G_{3} \preccurlyeq G_{4} \preccurlyeq G_{5}=G(\alpha, \beta)
$$

with no two equally large. Without loss of generality (by passing to subgroups of finite index) we may assume that each $G_{i}$ is an epimorphic image of $G_{i+1}$. Now $G(\alpha, \beta)$ is an extension of a finitely generated group by a copy of $\mathbf{Z}\left(p^{\infty}\right)$. If we denote by $\mathscr{C}$ the class of abelian groups which are either finitely generated or an extension of a finitely generated group by a copy of $\mathbf{Z}\left(p^{\infty}\right)$, then it is routine (see Fuchs $(1970,1973)$ or Kaplansky (1969) for the basic theory of abelian groups) to check that:

(1) $\mathscr{C}$ is closed under subgroups and epimorphic images;

(2) a torsion group in $\mathscr{C}$ is either finite or the direct sum of a finite group and a copy of $\mathbf{Z}\left(p^{\infty}\right)$

(3) an arbitrary group in $\mathscr{C}$ is either the direct sum of a finite group and a torsion-free group or the direct sum of a finite group, a copy of $\mathbf{Z}\left(p^{\infty}\right)$ and a finitely generated torsion-free group.

Hence, without loss of generality, we may further assume that each $G_{i}$ is either torsion-free or the direct sum of a copy of $\mathbf{Z}\left(p^{\infty}\right)$ and a finitely generated torsion-free group. Now consider the kernels $K_{i+1}$ of the epimorphisms from $G_{i+1}$ onto $G_{i}$. Since $G_{5}$ has torsion-free rank three and, whenever $K_{i+1}$ is not torsion, the torsionfree rank of $G_{i}$ is strictly less than that of $G_{i+1}$, we must have $K_{i+1}$ torsion for at least one $i$. Let $i_{0}$ be the biggest such $i$. Then $G_{i_{0}+1}$ must be of the form $A \oplus B$ with $A$ a copy of $\mathbf{Z}\left(p^{\infty}\right)$ and $B$ finitely generated torsion-free. Hence $K_{i_{0}+1}=A$ (otherwise $G_{i_{0}+1} / K_{i_{0}+1}=G_{i_{0}}$ would be isomorphic to $G_{i_{0}+1}$, and $G_{i_{0}}$ is isomorphic to $B$ and hence is finitely generated torsion-free. This implies that $G_{i}$ is finitely generated torsion-free for all $i \leqslant i_{0}$. Hence $K_{i}$ is torsion-free for all $i \leqslant i_{0}$, so the torsionfree rank of $G_{1}$ must be zero, that is $G_{1}$ is torsion. But $G_{1}$ is also torsion-free, so we have obtained a contradiction (remember $G_{1}$ is infinite). This concludes the proof of (a).

(b) Let $n$ be an integer. Then the automorphism $x \oplus y \oplus z \mapsto x \oplus(y+n z) \oplus z$ of $p^{-\infty} \mathbf{Z} \oplus p^{-\infty} \mathbf{Z} \oplus p^{-\infty} \mathbf{Z}$ is easily seen to restrict to an isomorphism from $G(\alpha, \beta)$ to $G(\alpha+n \beta, \beta)$. Hence, since $G(\alpha) \preccurlyeq G(\alpha, \beta)$, we have $G(\alpha+n \beta) \preccurlyeq G(\alpha, \beta)$ for all $n$. It remains to be shown that, when $\alpha$ and $\beta$ are algebraically independent over $Q$, $G(\alpha+m \beta)$ and $G(\alpha+n \beta)$ cannot be equally large unless $m=n$.

Now if $G$ and $H$ are torsion-free abelian groups of finite rank which are equally large then it must be the case that they have isomorphic subgroups of finite index. Then $G$ and $H$ are said to be quasi-isomorphic. A very special case of a result of Beaumont and Pierce (1961), Corollary 4.14, implies that, if $G\left(\alpha_{1}\right)$ and $G\left(\alpha_{2}\right)$ are quasi-isomorphic, then $\alpha_{2}$ is a rational (in fact linear fractional) function of $\alpha_{1}$. This can be seen directly as follows: If $G\left(\alpha_{1}\right)$ and $G\left(\alpha_{2}\right)$ have isomorphic subgroups 
$A_{1}$ and $A_{2}$, where $A_{1}$ has index $k$ in $G\left(\alpha_{1}\right)$, then the isomorphism restricts to an isomorphism from $k G\left(\alpha_{1}\right) \subseteq A_{1}$ to a subgroup of finite index in $G\left(\alpha_{2}\right)$. But $k G\left(\alpha_{1}\right)$ is isomorphic to $G\left(\alpha_{1}\right)$, so in fact there is an isomorphism $\varphi$ from $G\left(\alpha_{1}\right)$ to a subgroup of $G\left(\alpha_{2}\right)$. This isomorphism $\varphi$ extends to an automorphism of $\mathbf{Q} \oplus \mathbf{Q}$ (which is the divisible hull of $G\left(\alpha_{1}\right)$ and of $\left.G\left(\alpha_{2}\right)\right)$, so we have $\varphi(x \oplus y)=(a x+b y) \oplus(c x+d y)$ for some $a, b, c, d$ in Q. Since $\varphi$ carries $G\left(\alpha_{1}\right)$ into $G\left(\alpha_{2}\right)$ we conclude that, for all $x, y$ in $p^{-\infty} \mathbf{Z}$ with $\alpha_{1} x-y$ in $Z_{p}$, it must be the case that

$$
\alpha_{2}(a x+b y)-(c x+d y)=\left(a \alpha_{2}-c\right) x-\left(-b \alpha_{2}+d\right) y
$$

lies in $\mathbf{Z}_{p}$. This is easily seen to imply that $\alpha_{1}=\left(a \alpha_{2}-c\right) /\left(-b \alpha_{2}+d\right)$.

If $\alpha$ and $\beta$ are algebraically independent over $Q$ then, when $m \neq n$, no such linear fractional relation can hold between $\alpha_{1}=\alpha+m \beta$ and $\alpha_{2}=\alpha+n \beta$. Hence $G(\alpha+m \beta)$ and $G(\alpha+n \beta)$ cannot be equally large, so the proof of $(b)$ is finished.

\section{Conclusion}

Of the various questions suggested by the above example, perhaps the most natural is the following: Does there exist a group $G$ of height three such that there are infinitely many (up to $\simeq$ ) $H$ with $H \preccurlyeq G$ ? It can be shown that no abelian group $G$ with these properties exists.

\section{References}

R. A. Beaumont and R. S. Pierce (1961), 'Torsion-free groups of rank two', Mem. Amer. Math Soc. 38.

L. Fuchs (1970, 1973), Infinite abelian groups, Vols. I and II (Academic Press, New York).

I. Kaplansky (1969), Infinite abelian groups (University of Michigan Press, Ann Arbor, Michigan).

S. J. Pride (1976), 'The concept of "largeness" in group theory', Proc. Conf. on Word and Decision Problems in Algebra and Group Theory, Oxford, 1976 (to appear).

S. J. Pride (1979), 'On groups of finite height', J. Austral. Math. Soc. (Series A) 28, 87-99.

Department of Mathematics

University of California, Los Angeles

U.S.A. 